\title{
ALTERAÇÕES NA DISTRIBUIÇÃO DE POTÊNCIA CORTICAL EM FUNÇÃO DA CONSOLIDAÇÃO DA MEMÓRIA NO APRENDIZADO DE DATILOGRAFIA
}

\author{
Marlo Cunha', Victor Hugo Bastos², Heloisa Veiga ${ }^{3}$, Maurício Cagy $^{4}$, \\ Kaleb McDowell ${ }^{5}$, Vernon Furtado ${ }^{6}$, Roberto Piedade ${ }^{7}$, Pedro Ribeiro ${ }^{8}$
}

\begin{abstract}
RESUMO - O objetivo do presente estudo foi investigar alterações nos padrões eletroencefalográficos de sujeitos normais e destros durante o aprendizado de uma tarefa motora (datilografia). Estudos têm demonstrado que o córtex cerebral é susceptível a modificações durante a aprendizagem e que alterações nos padrões elétricos corticais ocorrem em função da aquisição de uma habilidade motora e da consolidação da memória. Assim, a atividade elétrica cortical dos sujeitos foi analisada antes e depois da prática motora. Os dados foram captados pelo "Braintech" 3000 e analisados pelo programa "Neurometrics". Para a análise estatística, variáveis comportamentais tais como tempo e erro, foram analisadas através do teste ANOVA "one-way" (diferenças entre blocos). Em seguida, foi utilizado um Teste-t pareado para os pares de eletrodos CZ-C3/CZ-C4, nas bandas de freqüência teta e alfa. Os resultados principais demonstraram mudança na performance através das variáveis comportamentais "tempo" e "número de erros". Concomitantemente, não foram observadas alterações na variável neurofisiológica "Potência Absoluta" na banda teta. Em contrapartida, houve um aumento significativo na banda alfa em áreas centrais (CZ-C3/CZ-C4). Tais resultados sugerem uma adaptação do córtex sensório-motor em função do treinamento de datilografia.
\end{abstract}

PALAVRAS-CHAVE: integração sensório-motora, memória de procedimento, EEG.

\begin{abstract}
Changes in cortical power distribution produced by memory consolidation as a function of a typewriting skill

ABSTRACT - The present study aimed to investigate alterations in EEG patterns in normal, right-handed individuals, during the process of learning a specific motor skill (typewriting). Recent studies have shown that the cerebral cortex is susceptible to several changes during a learning process and that alterations in the brain's electrical patterns take place as a result of the acquisition of a motor skill and memory consolidation. In this context, subjects' brain electrical activity was analyzed before and after the motor task. EEG data were collected by a Braintech 3000 and analyzed by Neurometrics. For the statistical analysis, the behavioral variables "time" and "number of errors" were assessed by a one-way ANOVA. For the neurophysiological variable "Absolute Power", a paired t-Test was performed for each pair of electrodes CZ-C3/CZ$\mathrm{C4}$, in the theta and alpha frequency bands. The main results demonstrated a change in performance, through both behavioral variables ("time" and "number of errors"). At the same time, no changes were observed for the neurophysiological variable ("Absolute Power") in the theta band. On the other hand, a significant increase was observed in the alpha band in central areas (CZ-C3/CZ-C4). These results suggest an adaptation of the sensory-motor cortex, as a consequence of the typewriting training.
\end{abstract}

KEY WORDS: motor-sensory integration, procedure memory, EEG.

Aprendizagem e memória são processos correlatos e indissociáveis. Aprendizagem corresponde à aqusição de novos conhecimentos e conseqüente modificação do comportamento, enquanto a memória pode ser entendida como a retenção deste conhecimento'. Desta forma, esses dois processos

\footnotetext{
Laboratório de Mapeamento Cerebral e Integração Sensório-Motora, Instituto de Psiquiatria (IPUB); Universidade Federal do Rio de Janeiro (UFRJ); Universidade Castelo Branco (PROCIHM); Department of Kinesiology and Program in Neuroscience and Cognitive Science, University of Maryland, College Park, USA (UM-USA): ${ }^{1}$ Mestrando, Laboratório de Mapeamento Cerebral e Integração SensórioMotora, IPUB - UFRJ; ${ }^{2}$ Mestre em Ciência da Motricidade Humana, PROCIHM; ${ }^{3}$ Mestranda, Laboratório de Mapeamento Cerebral e Integração Sensório-Motora, IPUB - UFRJ; ${ }^{4}$ D.Sc. em Engenharia Biomédica, UFRJ; ${ }^{5}$ PhD, UM-USA. ${ }^{6}$ Professor Titular, PROCIHM; 7Professor Adjunto III Doutor, IPUB, Coordenador do Laboratório de Mapeamento Cerebral e Integração Sensório-Motora, UFRJ; ${ }^{8}$ Professor Adjunto II PhD, Escola de Educação Física e Desportos, (IPUB), Laboratório de Mapeamento Cerebral e Integração Sensório-Motora, UFRJ; Professor Pesquisador, (PROCIHM).
}

Recebido 24 Novembro 2003, recebido na forma final 30 Janeiro 2004. Aceito 12 Março 2004. 
compartilham mecanismos neurais similares, sendo estes responsáveis também pela atenção, integração sensorial e percepção ${ }^{2}$. Não data de hoje o interesse de pesquisadores por alterações produzidas no sistema nervoso em função da aprendizagem motora, também conhecida como memória de procedimentos. Este tipo de memória é resultante do aumento da performance e proporcionalmente do incremento da precisão do gesto motor ${ }^{3}$. Com base na mesma definição, o lobo frontal (ambos os hemisférios) é apontado como estrutura importante para o aprendizado de procedimentos unilaterais. De forma complementar, a porção anterior do corpo caloso, por conectar esses dois hemisférios, torna-se crucial para a integração e transferência das habilidades de procedimento. Esse complexo estrutural proporciona base para o aprendizado de tarefas bimanuais. $\mathrm{O}$ aprendizado gradualmente produz diminuição no erro embutido na tarefa, aumento da coordenação e maior agilidade e velocidade na execução do movimento ${ }^{4}$. Esse implemento da performance, observado no processo de aprendizagem, tem sido associado a complexos mecanismos de consolidação de memória de longo prazo.

Em função da diversidade de componentes que são reorganizados plasticamente, o entendimento dos efeitos da aprendizagem motora no córtex tornou-se um desafio no campo das neurociências. Acomodações plásticas neurais do sistema nervoso têm norteado a compreensão de modelos experimentais sobre o arquivamento de informações motoras. A combinação entre memória sensorial (estímulos sensoriais), memória de curto prazo (memória de trabalho) e memória de longa duração representada no sistema nervoso através da consolidação e a execução do gesto motor levaria a um novo ordenamento na configuração neural ${ }^{5,6}$. Em especial durante o aprendizado de uma tarefa motora, essa representação interna produziria incremento na eficácia ("força") sináptica de neurônios em áreas corticais e sub-corticais? . A elaboração de um modelo interno, a partir da aprendizagem motora, sustenta-se na conectividade e organização de uma nova rede neural ${ }^{8}$. O ajuste dessa nova arquitetura neural produziria um sistema de referência no qual o gesto motor tornar-se-ia mais eficiente com o aumento da experiência?.

Dentro deste contexto, o objetivo deste estudo é investigar a influência do aprendizado de uma tarefa motora nesta nova organização dos mapas corticais. Experimento recente evidenciou que a incorporação do gesto motor a partir da repetição produziria alterações neuronais capazes de serem detectadas com o uso da eletroencefalografia quantitativa $(E E G q){ }^{10}$. Neste mesmo estudo, é relatado aumento nas bandas de freqüência teta e alfa, em áreas frontais e sobre o córtex somato-sensorial, respectivamente, em função da prática motora. Adicionalmente, é sugerida redução da atividade elétrica em áreas corticais não necessárias à tarefa, em paralelo ao aumento dos níveis de habilidade do sujeito. A eletroencefalografia (EEG) tem sido amplamente utilizada na avaliação de mudanças eletrofisiológicas relacionadas a diferentes desordens psiquiátricas. A sensibilidade do EEG em detectar correlatos de desordens psiquiátricas pode ser incrementada através de métodos de análise quantitativa (EEGq) ${ }^{11-14}$. O EEGq, especificamente, se tornou ao longo dos últimos anos ferramenta de grande utilidade clínica na área da neurociência comportamental.

\section{MÉTODO}

Amostra - A amostra do estudo consistiu de 29 sujeitos, sendo 14 do sexo masculino e 15 do feminino, entre 20 e 40 anos. Os sujeitos, escolhidos dentre os alunos de Graduação e Mestrado da Universidade Castelo Branco (UCB), não apresentavam qualquer tipo de comprometimento de saúde física ou mental, eram sadios, livres de qualquer déficit cognitivo e não faziam, uso de substâncias psicotrópicas ou psicoativas. Foi aplicado um questionário detalhado para identificar e excluir do experimento qualquer sujeito que pudesse contaminar futuros resultados. Os indivíduos não tinham experiência prévia em datilografia e a lateralidade foi usada como critério de exclusão. Para tal, foi aplicado o inventário de Edinburgh ${ }^{15}$ a fim de verificar a predominância dos participantes (destro versus sinistro). Indivíduos com predominância da mão esquerda (sinistro) foram, conseqüentemente, eliminados do experimento. Os sujeitos assinaram declaração de consentimento na qual foi descrita, em detalhes, a condição experimental. O experimento foi submetido à Comissão de Ética da UCB para aprovação.

Procedimento experimental - A sala utilizada para captação do sinal eletroencefalográfico foi preparada para isolamento de som e, durante a aquisição dos dados, as luzes da sala foram reduzidas. Os sujeitos sentaram-se confortavelmente em cadeira com suporte para os braços, no intuito de minimizar artefatos musculares. A cadeira dos participantes ficou a uma distância de aproximadamente $40 \mathrm{~cm}$ da mesa, variando de acordo com o comprimento do antebraço de cada indivíduo. Uma máquina de datilografia, estilo antigo (marca: Olivetti Linea 98), foi colocada sobre a mesa onde foi realizada a tarefa motora. O teclado da máquina foi coberto com uma "caixa de madeira", a fim de evitar que os participantes tivessem informação visual da posição das mãos, 
obrigando-os a criar uma "referência espacial" para o teclado. A tarefa consistiu na utilização de um método de datilografia, Celso Santos ${ }^{16}$, de aprendizagem progressiva, cujo treinamento foi realizado em um único dia. Este método vem sendo usado e aprovado como eficiente durante anos em diferentes cursos de datilografia. 0 método consiste de exercícios que expõem o aprendiz, inicialmente, a gestos mais simples que aumentam progressivamente no grau de complexidade, permitindo, assim, que o indivíduo amplie, gradativamente, destreza e coordenação bi-manual. As lições foram afixadas em quadro de cortiça colocado na parede em frente aos participantes e, à medida que o indivíduo terminava, a lição seguinte era firmada no quadro. Cada bloco de exercícios consistiu de dez colunas e doze linhas (matriz $10 \times 12$ ), sendo cada participante obrigado a realizar quatro blocos desta matriz para completar uma lição. Os participantes foram instruídos a realizarem a tarefa da forma mais rápida e eficiente possível. O tempo total de realização dos quatro blocos e de cada bloco individualmente foi mensurado. Desta forma, seria possível verificar se houve ou não melhora progressiva de desempenho entre blocos. O número de erros foi computado, especificamente, em cada bloco e no total deles. Sendo assim, a melhora progressiva do gesto motor e a diminuição do número de erros foram estimadas. No presente experimento, os sujeitos executaram a técnica de datilografia por uma ho$\mathrm{ra}$, descansaram por vinte minutos e realizaram mais uma hora da tarefa motora (duas horas no total). No final de cada hora, se o exercício não tivesse sido terminado, ele não era considerado. Esta regra se fez necessária a fim de que cada sujeito tivesse uma hora exata em cada período de treinamento. Por fim, folhas de papel A4 (210 x $297 \mathrm{~mm}$ ) foram colocadas na máquina de datilografia antes do início da tarefa. Os participantes foram instruídos a executarem dois blocos por folha. No final do segundo bloco, a folha foi substituída por outra.

Aquisição de dados - Para a captação do sinal eletroencefalográfico, foi utilizado o aparelho Braintech 3000 (Emsa - Instrumentos Médicos, Brazil), sistema que utiliza uma placa conversora analógica-digital (A/D) de 32 canais com resolução de 12 bits, colocada em um slote ISA de um Pentium III, com um processador de $750 \mathrm{~Hz}$. Os sinais eletrofisiológicos foram filtrados entre 0,01 (passabaixas) e $100 \mathrm{~Hz}$ (passa-altas), tendo uma taxa de amostragem de $200 \mathrm{~Hz}$. Foi utilizado o software de aquisição denominado EEG Captação (Emsa-Delphi 5.0), com um filtro Notch de $60 \mathrm{~Hz}$, e ainda filtros de corte de $0,3 \mathrm{~Hz}$ (passa-altas) e $25 \mathrm{~Hz}$ (passa-baixas). Foi usado o sistema internacional 10/2017 para a colocação de 19 eletrodos monopolares ao longo do escalpo (áreas: frontal, temporal, parietal e occipital) e um eletrodo em cada orelha (lóbulo). Os eletrodos foram montados em uma touca de nylon (ElectroCap Inc., Fairfax, VA) com o sistema 1020 pré-fixado. Este sistema se refere a um padrão de colocação de eletrodos estabelecido internacionalmen- te, que utiliza marcas anatômicas para demarcar a colocação e a distância entre eletrodos. A touca foi colocada e ajustada individualmente em cada participante, obedecendo à circunferência da cabeça (toucas de tamanhos variados). Os lóbulos das orelhas foram usados como referência (bi-auricular). O sinal adquirido em um determinado eletrodo é resultante da diferença entre o potencial elétrico do mesmo no escalpo e a referência pré-estabelecida. Foram verificados "a priori" os níveis de impedância de cada eletrodo, cujos valores deveriam estar entre $5-10 \mathrm{~K}$ ohms $(\Omega)$ e ser mantidos nesses padrões. Os sinais adquiridos deveriam estar com o total de amplitude (pico a pico) menor que $100 \mu \mathrm{V}$. Por esse motivo, o sinal foi amplificado com ganhos variando de 20000. Os sinais eletroencefalográficos adquiridos flutuaram entre 0,01 e $50 \mathrm{~Hz}$. A atividade elétrica ocular foi estimada com a colocação de dois eletrodos de $9 \mathrm{~mm}$ de diâmetro montados de forma bipolar. Os eletrodos foram posicionados, respectivamente, acima e abaixo da órbita do olho direito para registrar movimentos oculares verticais, e no canto externo do mesmo para registrar movimentos oculares horizontais. Artefatos visuais foram inspecionados com a utilização de um programa de visualização denominado EEG Telas (Emsa-Delphi 5.0).

Análise de dados e cálculo das variáveis dependentes - No experimento, foram analisados dois tipos de variáveis: comportamentais e neurofisiológicas, a última sendo extraída da EEGq. As variáveis comportamentais que mensuram performance foram dadas através do tempo de execução dos blocos e número de erros em cada bloco. Após a coleta dos dados e respectivo arquivamento, foram computadas análises para extração das variáveis dependentes. Após a inspeção visual para a retirada de possíveis artefatos, os sinais eletroencefalográficos foram processados por software denominado Neurometrics (NxLink, Ltd., USA), o qual extraiu dos dados, ou seja, das séries temporais, a variável neurofisiológica relevante ao experimento: distribuição de Potência Absoluta em eletrodos homólogos posicionados em hemisférios diferentes (direito x esquerdo).

Potência absoluta - Potência é uma medida de amplitude: quanto maior a amplitude, maior a quantidade de potência no sinal eletroencefalográfico. A Potência Absoluta é expressa em picowatts $\left(\mu \mathrm{V}^{2}\right)$ e reflete a quantidade de energia presente em uma dada banda de freqüência, em um par específico de eletrodos. O Neurometrics analisa a distribuição de potência no escalpo. Através deste programa, é aferida a variação de potência entre pares de eletrodos.

Análise estatística - Devido ao fato de ocuparem os eletrodos posição espacial diferenciada no escalpo, optou-se por uma análise estatística independente. Nas variáveis comportamentais, optou-se por análise de variância (one-way) com o intuito de identificar as diferenças en- 
tre os 4 blocos do exercício 1. Os dados do EEG foram mensurados em dois momentos diferentes: antes e depois do início da tarefa (datilografia). A fim de avaliar se o aprendizado de datilografia produz alterações significativas na Potência Absoluta, foi utilizado o Teste-t para cada par de eletrodos (CZ/C3-CZ/C4), nas bandas teta e alfa.

\section{RESULTADOS}

Os resultados foram divididos em duas formas de variáveis dependentes: comportamentais e neurofisiológicas. As variáveis comportamentais foram voltadas para a caracterização da performance; as neurofisiológicas, para as alterações plásticas neurocorticais.

Variáveis comportamentais - A Figura 1 descreve a variação do tempo de execução em cada um dos
4 blocos. Análise estatística demonstrou diferença significativa entre os 4 blocos $(p \sim 0)$, análises posthoc indicaram diferença entre o bloco 1 e os demais $(p=0,003)$ e diferença entre os blocos 2 e 4 ( $p$ $=0,009$ ). Não foram detectadas diferenças entre os blocos 2 e $3(p=0,112)$ e 3 e $4(0,292)$. Desta forma, evidenciou-se modificação entre o bloco 1 (média $=13,52 /$ desv. padrão $=5,27$ ) e o bloco 2 (média $=$ $10,38 /$ desv. padrão $=4,52$ ).

Neste contexto, a Figura 2 expressa o número de erros dentro de cada bloco do exercício 1. Resultados estatísticos demonstraram diferença significativa entre os 4 blocos $(p \sim 0)$. A avaliação post-hoc evidenciou diferença entre o bloco 1 e os demais $(p=0,005)$. Nenhuma diferença foi detectada em

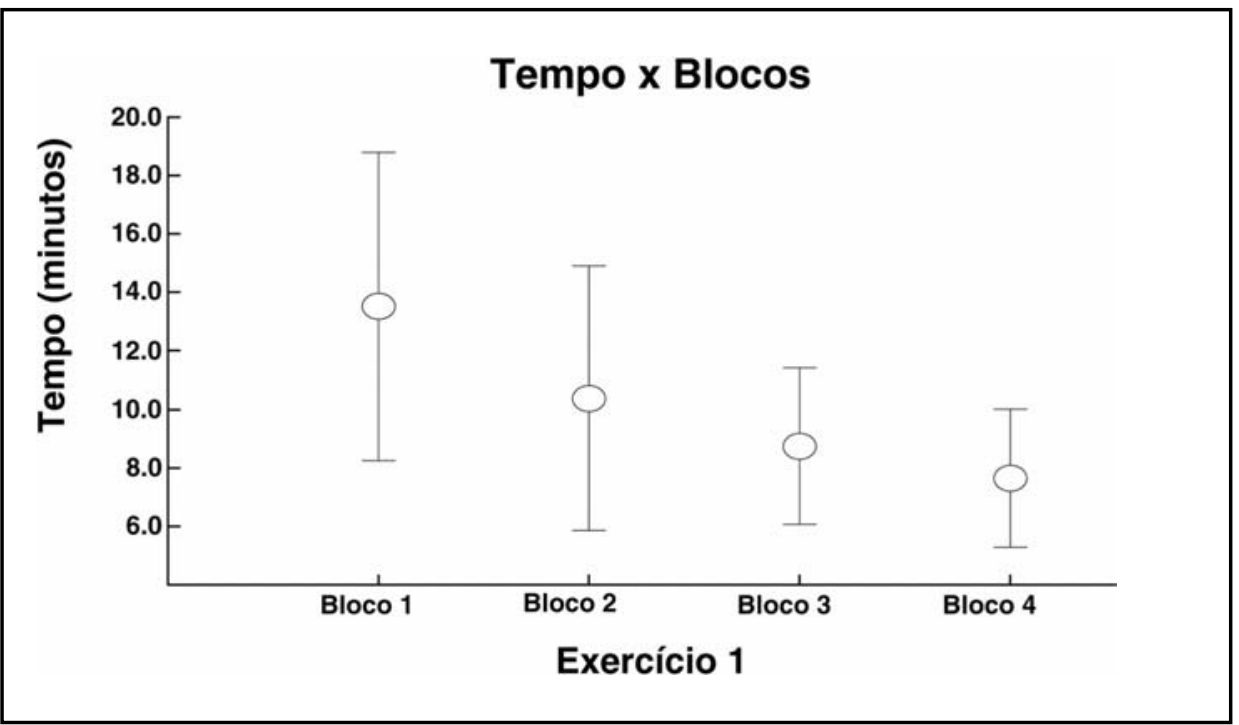

Fig 1. Relação entre tempo de execução da tarefa e blocos.

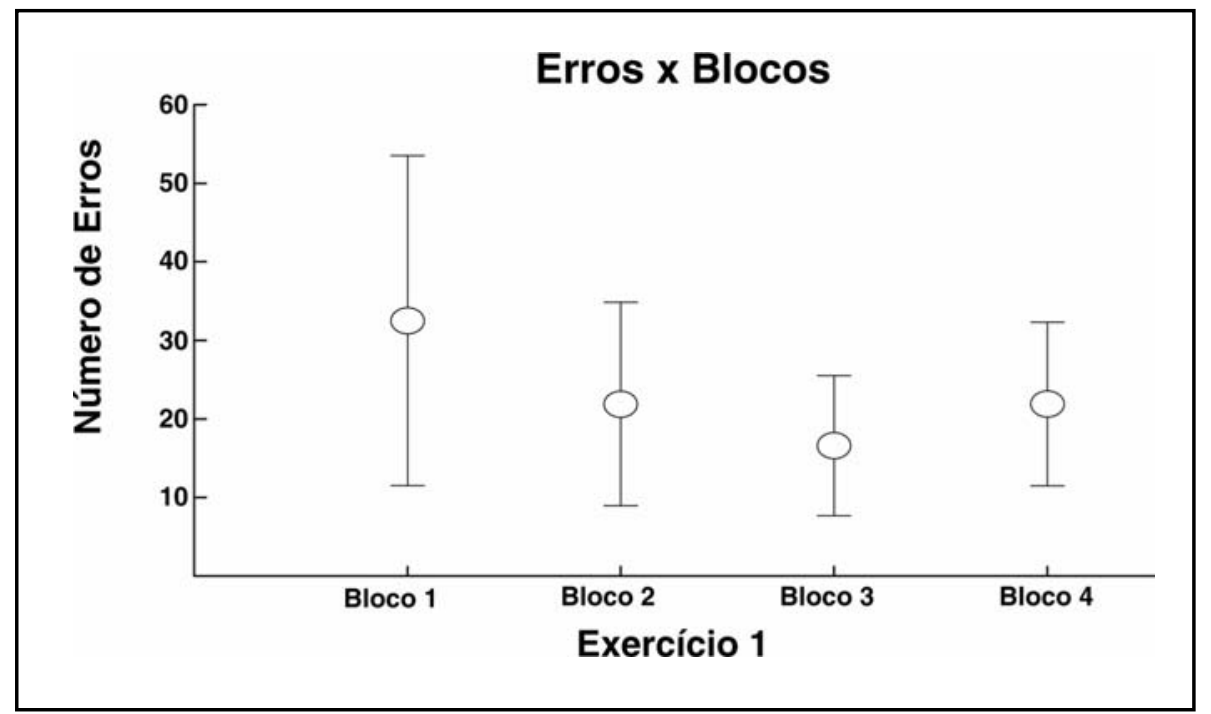

Fig 2. Relação entre número de erros e blocos. 


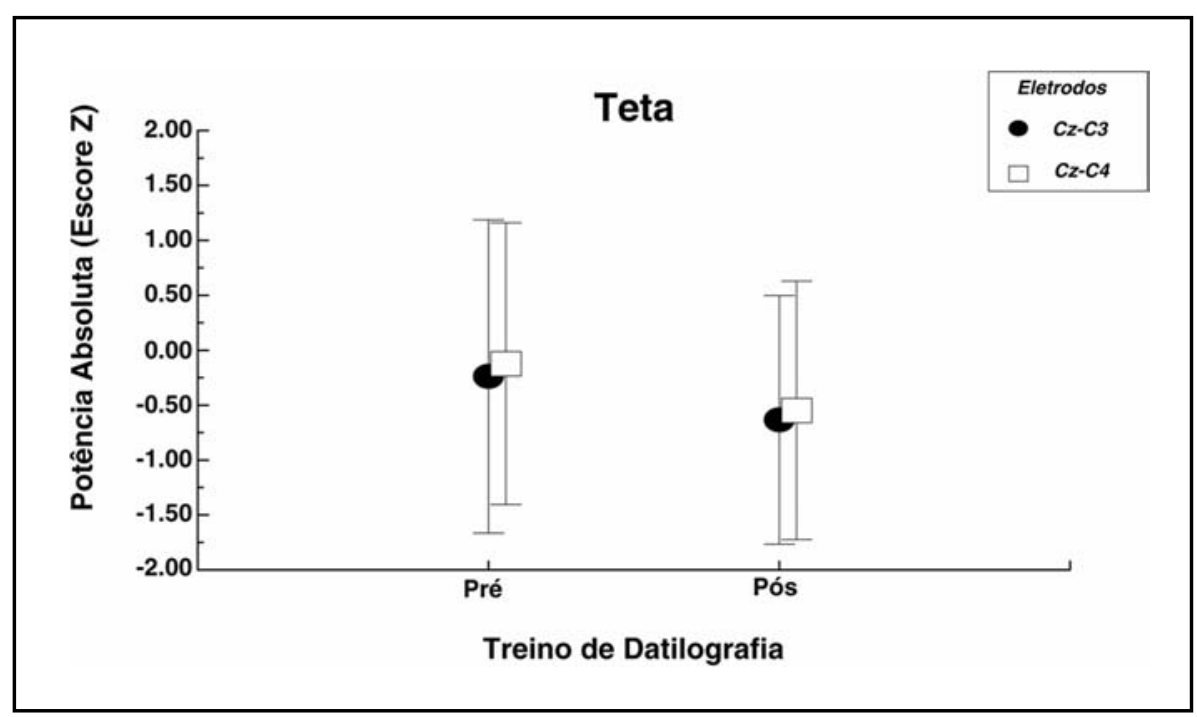

Fig 3. Variação da potência em função do treinamento de datilografia.

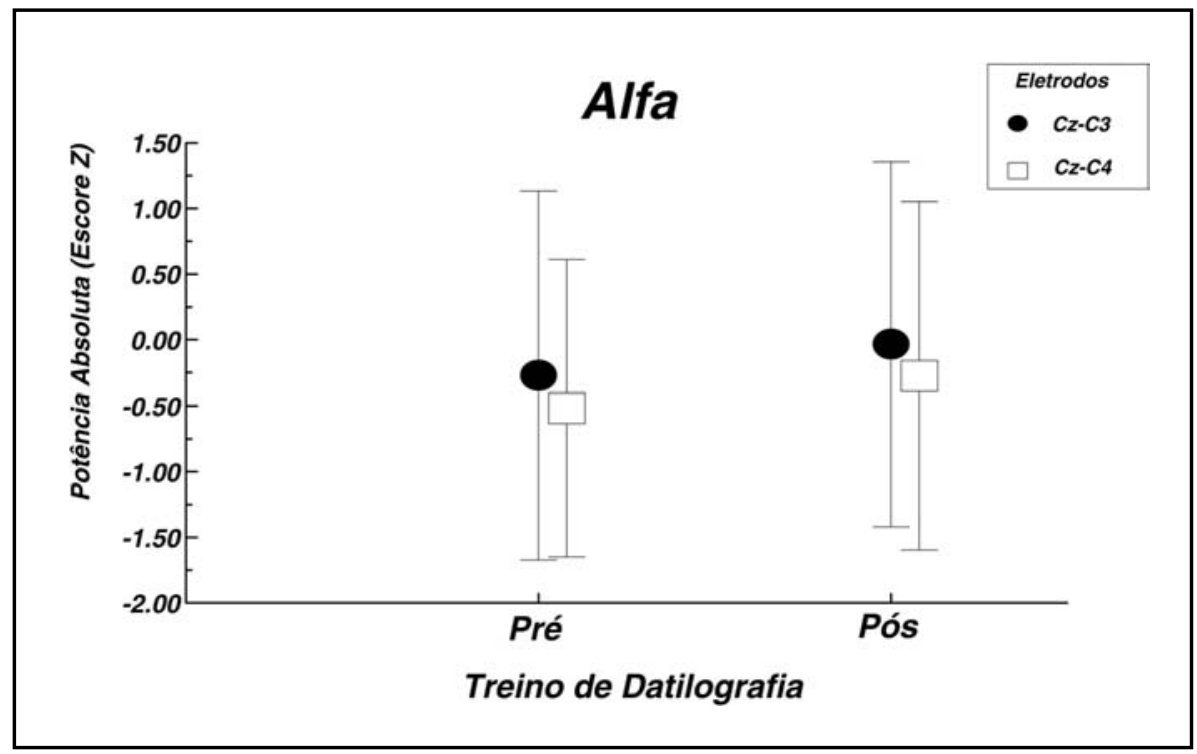

Fig 4. Variação da potência em função do treinamento de datilografia.

relação aos outros blocos. Sendo assim, a principal diferença ocorreu entre o bloco 1 e os demais, marcando a importância no primeiro bloco do começo do aprendizado.

Variáveis neurofisiológicas - A Figura 3 descreve a variação da Potência Absoluta entre o pré e o póstreinamento nas regiões CZ/C3 e CZ/C4 na banda teta. Análise estatística não demonstrou diferença significativa entre as duas condições $(p=0,525)$ para ambos os pares de eletrodos.

A Figura 4 descreve a variação da Potência Absoluta entre o pré e o pós-treinamento nas regiões CZ/C3 e CZ/C4 na banda alfa. Análise estatística de- monstrou diferença significativa entre as duas condições $(p=0,022 / 0,036)$ para ambos os pares de eletrodos.

\section{DISCUSSÃO}

A presente investigação se propôs a observar mudanças corticais a partir da exposição de sujeitos a uma tarefa motora (datilografar). Especificamente, foram observadas modificações neurais que ocorrem durante a aquisição de uma habilidade sensoriomotora envolvendo memória sensorial (localização espacial dos dedos em relação ao teclado) e memória de curto prazo. Neste contexto, o EEGq foi usado para analisar tais modificações. Primeira- 
mente, as variáveis comportamentais, tempo $\mathrm{x}$ erros, indicaram que a prática de datilografia melhorou a habilidade dos sujeitos para executar a tarefa motora. Os resultados apontaram diminuição no tempo, especialmente quando o primeiro bloco foi comparado aos outros análise (post-hoc). Em outras palavras, com o aumento da performance, a quantidade de tempo necessário para terminar o segundo, terceiro, e quarto blocos diminuiu consideravelmente. A diminuição do tempo de execução da tarefa entre blocos caracteriza o processo contínuo de aprendizagem.

O número de erros também diminuiu à medida que os sujeitos ficaram mais hábeis na tarefa de datilografia. Essencialmente, este padrão de resultados foi evidenciado entre o primeiro bloco e os demais. Sendo assim, os resultados presentes reproduziram achados anteriores, nos quais foi percebido aumento do desempenho quando indivíduos foram expostos a uma tarefa sensoriomotora ${ }^{4,10}$. As melhorias no desempenho (tempo x erro) estão de acordo com achados anteriores de várias pesquisas sensoriomotoras, nas quais sujeitos tiveram que administrar informação da memória processual ${ }^{18}$. Além disso, baseado nos resultados presentes, foi claramente caracterizado um "período crítico" durante o processo de aprendizagem, na transição entre o primeiro e o segundo bloco, em ambas as variáveis (tempo x erro). Estes achados sugerem que este "momento crítico" está associado à transição entre os mecanismos de controle e a automação do movimento. Mecanismos de controle estão associados à fase inicial do aprendizado, quando os indivíduos necessitam alocar uma dose excessiva de atenção ao desempenhar o gesto motor. Em contrapartida, uma vez automatizada a tarefa, este excesso de atenção e dedicação não se faz necessário.

Em relação às medidas neurofisiológicas, o EEGq auxilia a monitoração das mudanças cerebrais que ocorrem quando se executa uma atividade sensoriomotora. $\mathrm{O}$ acompanhamento das modificações dos sinais do EEG pode ser correlacionado com a reorganização neural, que é intrínseca à construção de habilidades motoras complexas. Apesar da baixa resolução espacial do EEG, tais dados apresentam excelente capacidade temporal. Assim, a repetição imposta pelo período de treinamento pode ser observada através dos padrões de EEG.

Na presente estudo, as bandas de freqüências teta $(4,0-7,5 \mathrm{~Hz})$ e alfa $(8-12 \mathrm{~Hz})$ foram usadas para avaliar atenção e mudanças cognitivas produzidas pela atividade de datilografia. A medida de Potência Absoluta foi utilizada com a finalidade de observar possíveis alterações corticais. Potência Absoluta expressa a quantidade de energia presente em uma banda, desconsiderando todas as outras freqüências embutidas no espectro. Neste contexto, os presentes resultados mostraram diminuição da Potência Absoluta entre o pré e o póstreinamento na banda teta, embora sem diferença estatística. Tal diminuição foi encontrada em eletrodos centrais (CZ-C3)/(CZ-C4). Uma possível interpretação desta observação é o fato de que mudanças na banda teta representam diferentes quantidades de atenção alocadas no princípio da tarefa comparadas ao fim do segundo bloco. $O$ presente achado contrapõe-se a outros experimentos, cujos resultados indicaram incremento de teta com o aperfeiçoamento da tarefa ${ }^{10}$. Estas modificações em regiões centrais (CZ-C3)/(CZ-C4), em particular, representam o envolvimento da área motora suplementar (SMA), córtex pré-motor (PME) e córtex somato-sensorial (SMC) em processos que envolvem atenção.

Por outro lado, foram encontradas diferenças significativas nos mesmos eletrodos na faixa alfa. Foi verificado incremento entre o pré e pós-tratamento durante o experimento. Este aumento da Potência Absoluta (banda alfa) entre a linha de base (pré-tratamento) e a segunda medida sugere uma consolidação do aprendizado e da memória de longo prazo ${ }^{1}$. A presença de ritmos alfa reflete atenuação da atividade neuronal cortical. Conseqüentemente, a amplitude deste ritmo é inversamente proporcional à atividade de uma determinada população neuronal pré-determinada em uma dada região cortical. Neste contexto, aumento de Potência Absoluta na banda alfa após o aprendizado motor pode ser interpretado como redução de atividade de neurônios na região especificada.

\section{CONCLUSÃO}

A partir dos achados do presente estudo, podemos concluir que a distribuição de potência no córtex cerebral pode ser utilizada como um indicador da consolidação da memória e do aprendizado. Neste contexto, abre-se caminho para novos experimentos que combinem diferentes psicofármacos e populações específicas (psiquiátricas, neurológicas, entre outras). Por exemplo, já foram demonstrados efeitos de drogas de ação colinérgica na atenção visual sustentada ${ }^{19} \mathrm{e}$ ainda efeitos produzidos por benzodiazepínicos nos processos de inibição 
pós-sináptica (GABA-A) ${ }^{20}$, utilizando tarefas motoras (movimentos de apreensão de objetos) combinadas a medidas espectrais do EEG. Desta forma, será possível observar os efeitos de medicamentos que afetam o sistema nervoso central e de diferentes doenças na atividade elétrica cerebral. Especificamente, o uso de tarefas motoras associadas a medidas eletroencefalográficas vem sendo explorado no entendimento dos mecanismos de desordens que afetam os núcleos da base, particularmente na doença de Parkinson ${ }^{21}$. Assim, será possível analisar as alterações no processo de aprendizagem e na consolidação da memória nestes pacientes.

\section{REFERÊNCIAS}

1. Maxwell J, Masters R, Eves F. The role of working memory in motor learning and performance. Conscious Cogn 2003;12:376-402.

2. Jueptner M, Stephan K, Frith C, Brooks D, Frackowiak R, Passingham R. Anatomy of motor learning: I. Frontal cortex and attention to action. J Neurophysiol 1997;77:1313-1324.

3. Guise E, del Pesce M, Foschi N, Quattrini A, Papo I, Lassonde M. Collosal and cortical contribution to procedural learning. Brain 1999;122:10491062.

4. Karni A, Gundela M, Jezzard P, Adams M, Turner R, Ungerlder L. Functional MRI evidence for adult motor cortex plasticity during motor skill learning. Science 1995;377:155-158.

5. Cohen L, Brasil N, Pascual-Leone L, Hallet M. Plasticity of cortical motor output organization following deafferentation, cerebral lesions, and skill acquisition. Adv Neurol 1993;63:187-200.

6. Donoghue J. Plasticity of sensorimotor representations. Curr Opin Neurobiol 1995;5:749-754.
7. Gandolfo F, Li C, Benda B, Schioppa C, Bizzi E. Cortical correlates of learning in monkeys adapting to a new dynamical environment. Proc Natl Acad Sci 2000;29:2259-2263.

8. Gottlieb G. The generation of the efferent command and the importance of joint compliance in fast elbow movements. Exper Brain Res 1994; 97:545-550.

9. Wolpert D, Ghahramani Z, Flanagan R. Perspectives and problems in motor learning. Trends Cogn Sci 2001;5:487-494.

10. Smith M, McEvoy L, Gevins A. Neurophysiological indices of strategy development and skill acquisition. Cogn Brain Res 1999;7:389-404.

11. Fontenelle L, Piedade RA, Marques C, Menezes GB, Versiani M. Eletroencefalografia quantitativa no transtorno obsessivo-compulsivo: resultados preliminares. Arq Neuropsiquiatr 2000;58:677-682.

12. Anghinah R, Kanda PAM, Jorge MS, Lima EE, Pascuzzi I, Melo AC. Estudo da coerência do eletroencefalograma para a banda de freqüência alfa em indivíduos adultos normais e com provável demência do tipo Alzheimer. Arq Neuropsiquiatr 2000;58:272-275.

13. Veiga H, Deslandes A, Cagy M, Fiszman A, Piedade RA, Ribeiro P. Neurocortical electrical activity tomography in chronic schizophrenics. Arq Neuropsiquiatr 2003;61:712-717.

14. Dantas FG, Yacubian EM, Jorge CL, Pedreira CC, Bueno JF, Valério RM. Clinical and EEG analysis of mesial and lateral temporal lobe seizures. Arq Neuropsiquiatr 1998;56:341-349.

15. Oldfield R. The assessment and analysis of handedness: the Edinburgh inventory. Neuropsycologia 1971;9:97-113.

16. Santos C. Novíssimo guia do datilógrafo. 40 Ed. São Paulo: Saraiva, 1997.

17. Jasper $\mathrm{H}$. The ten-twenty electrode system of the international federation. EEG Clin Neurophysiol 1958;10:371-375.

18. Carlson R, Lundy D, Scheider W. Strategy guidance and memory aiding in learning a problem-solving skill. Hum Factors 1992;34:129-45.

19. Mumenthaler MS, Yesavage JA, Taylor JL, et al. Psychoactive drugs and pilot performance: a comparison of nicotine, donepezil, and alcohol effects. Neuropsychopharmacology 2003;28:1366-1373.

20. Baker MR, Baker SN. The effect of diazepam on motor cortical oscillations and corticomuscular coherence studied in man. J Physiol 2003;546:931-942.

21. Plat FM, Praamstra P, Horstink MW. Redundant-signals effects on reaction time, response force, and movement-related potentials in Parkinson's disease. Exp Brain Res 2000;130:533-539. 